\title{
In honor of James P. LeSage
}

\author{
Manfred M. Fischer
}

Received: 28 July 2011/Accepted: 5 August 2011/Published online: 26 August 2011

(C) Springer-Verlag 2011

One can have an impact on a field in a variety of ways such as papers published in prestigious journals, through service to associations, and through students. Certainly, James LeSage has published extensively in a variety of fields including economics and econometrics, regional science, spatial econometrics and statistics. He has helped organize sessions and performed other conference-related work, and he has involved students in the field of spatial econometrics. But his sincere desire to aid research and researchers in this field merits special mention. This takes a variety of forms, the most important being his presence on the Internet in the form of his econometrics toolbox that includes a substantial spatial component. He helps a large number of individuals interested in spatial research through his e-mail support of the toolbox and by answering general questions pertaining to spatial econometrics. This web site collection of public domain econometric and spatial econometric algorithms receives over 100,000 visits from individuals in over 100 countries during a typical 3-month period.

James LeSage has shown a range of intellectual interests since he began publishing in 1980. His early work over the period to about 1995 focused on econometric modeling of interregional labor markets, based on Bayesian variants of vector autoregressive and error correction models. These variants developed Bayesian priors that were being used at the time to model macroeconomic time series. Special features of interregional time series were incorporated into stochastic prior restrictions on the parameters. For example, co-integrating relationships between local and export employment that lead to statistical equilibrium relationships were exploited to improve forecasting performance for regional labor markets in articles that appeared in Review of Economics and Statistics, Journal of Regional

\footnotetext{
M. M. Fischer ( $₫)$

Institute for Economic Geography and GIScience,

Vienna University of Economics and Business, Nordbergstr. 15/4/A, 1090 Vienna, Austria

e-mail: Manfred.Fischer@wu.ac.at

URL: http://www.wu.ac.at/wgi/en
} 
Science, Regional Science and Urban Economics and International Regional Science Review.

Since the mid-1990s, James LeSage has focused more exclusively on spatial econometric methods and techniques, beginning with a 1997 publication of "Bayesian estimation of spatial autoregressive models", in International Regional Science Review. This work was the first to apply Markov chain Monte Carlo (MCMC) estimation methods to the family of spatial regression models introduced in Anselin's 1988 textbook. Other work regarding MCMC estimation of spatial regression models includes articles on probit and Tobit variants as well as origindestination flow variants of these models, and expository articles on Bayesian methods applied to spatial regression models that have appeared in the Journal of Regional Science, Geographical Analysis and as chapters in texts devoted to methodological developments in spatial econometrics.

His most recent work includes an article [co-authored with Kelley Pace] that appeared in Journal of Econometrics (2007) describing a matrix exponential approach to spatial regression. This spatial specification represents one of the few new approaches to these models since Ord's (1975) seminal work. Use of a matrix exponential spatial specification that replaces geometric decay over space with exponential decay is shown to eliminate the troublesome log-determinant term that appears in the likelihood function of conventional spatial regression models.

His recent work also covers many topics central to the application of spatial econometrics. For example, a co-authored paper published in Spatial Economic Analysis (2008) highlights some prominent aspects that are essential for anyone applying spatial econometrics. First, ignoring space by involving an inappropriate model specification will give the wrong answers, and wrongly interpreting the parameters of an appropriate model is equally likely to mislead. Second, an appropriate model in very many circumstances turns out to be the Spatial Durbin model that is characterized by endogenous and exogenous spatial lags. The presence of lagged variables leads to nuances with respect to the interpretation of the effects of variables, since spillovers cause both direct and indirect effects that need to be accounted for. A third dimension of the paper is provided by the application of Bayesian model averaging methods. While parameter uncertainty is well known, model uncertainty_-perhaps engendered by uncertainty regarding the true structure of the spatial weight matrix-is less explored, and the paper explains analytical techniques that can handle inference given model uncertainty.

During his career, James LeSage has published around 90 journal articles, 20 book chapters, and one path-breaking book, Introduction to Spatial Econometrics (coauthored with Kelley Pace) that raises the bar in spatial economics (Elhorst 2010).

With all these research accomplishments he has built up a world-class reputation. James LeSage is viewed as one of the intellectual leaders and most influential authors in regional science, in terms of published research output and citations (see Rey and Anselin 2000; Isserman 2004 for evidence). He received the 2009 Fellows Award for outstanding contributions and dedicated leadership to the field of Regional Science. Moreover he has been named in the "Applied Econometrics Hall 
of Fame", a list of 50 publishers worldwide of applied econometrics in 15 leading econometric journals (see Baltagi 1999).

It is our great pleasure and privilege to publish this special issue honoring James LeSage' prolific contributions to the field of spatial econometrics. The papers in this issue represent an array of topics that represent James LeSage's contributions to spatial econometrics. The first article, written by Paul Elhorst, provides an authoritative summary of the current state of the art in specification and estimation of dynamic spatial panel data models, a collection of models for spatial panels extended to include one or more of the following variables and/or error terms: a dependent variable lagged in space or time or in both, independent variables lagged in space or time, spatial error autocorrelation, serial error autocorrelation, spatialspecific and time-specific effects.

The second contribution in this issue, by Andrew Finley, Sudipto Banerjee and Alan E. Gelfand, shifts attention to a class of models where space is viewed as continuous and time is taken to be discrete. Scalability is achieved by using a lowrank predictive process to reduce the dimensionality of the data and ease the computational burden of estimating the spatiotemporal process of interest in the case of large data sets. The proposed models are illustrated using weather station data collected over the northeastern United States between 2000 and 2005. Here, the authors' interest is to use readily available predictors, association among measurements at a given station, as well as dependence across space and time to improve prediction for incomplete station records and locations where station data do not exist.

In the third paper, Olivier Parent compares alternative models that have been proposed in the panel data literature to provide a better understanding of how new ideas diffuse across space and time. The author analyzes the role of knowledge production and spatiotemporal spillover effects using a space-time panel data set covering 49 US states over the period 1994-2005. The objective is to test for existence of regional knowledge spillovers in the context of a space-time dynamic framework suggested by the knowledge production function. A space-time specification is set forth that can be applied to panel data models that include random effects. The results indicate that the space-time panel data are consistent with the presence of strong spatiotemporal regional spillovers of knowledge. The empirical findings are interpreted in light of the existing theoretical and empirical literature on endogenous growth.

The single spatial parameter in the spatial autoregressive model affects both the estimation of spillovers and the estimation of spatial disturbances. Consequently, the spatial autoregressive model has the undesirable property that if the degree of spatial dependence in the disturbances differs from that in the spillovers, neither may be estimated correctly. Kelley Pace and Shuang Zhu show theoretically that the dependence structure for the spillovers and disturbances can differ and conduct a Monte Carlo experiment that verifies these findings. In contrast, estimates from a simple separable model show little bias in all the scenarios. The authors demonstrate differences between the spatial autoregressive model and the separable model on five empirical examples. 
There is a common belief that the presence of residual spatial autocorrelation in OLS regression leads to inflated significance levels in beta coefficients and, in particular, inflated levels relative to the more efficient spatial error model. But simulations show that this is not always the case. The final contribution, written by Tony E. Smith and Ka Lok Lee, examines this question from a geometric viewpoint. The key idea is to characterize the OLS test statistic in terms of angle cosines and examine the geometric implications of this characterization. The authors show that if the independent variables in the regression exhibit no spatial autocorrelation, then the distribution of the test statistics for individual beta coefficients in OLS is independent of any spatial autocorrelation in the error term. Hence, inferences about betas exhibit all the optimality properties of the classic uncorrelated error case. But, a second more important series of results show that if spatial autocorrelation is present in both the dependent and independent variables, then the conventional wisdom is correct. In particular, even when an independent variable is statistically independent of the dependent variable, such joint spatial dependencies tend to produce spurious correlation that results in over-rejection of the null hypothesis.

\section{References}

Anselin L (1988) Spatial econometrics: Methods and models. Kluwer Academic Publishers, Dordrecht Baltagi BH (1999) Applied econometrics rankings: 1989-1995. J Appl Econ 14(4):423-441

Elhorst JP (2010) Applied spatial econometrics: Raising the bar. Spat Econ Anal 5(1):9-28

Isserman AA (2004) Intellectual leaders of regional science: a half-century citation study. Pap Reg Sci 83(1):91-126

LeSage JP (1990) A comparison of the forecasting ability of ECM and VAR models. Rev Econ Stat 72(4):664-671

LeSage JP (1997) Bayesian estimation of spatial autoregressive models. Int Reg Sci Rev 18(1):33-53

LeSage JP (2000) Bayesian estimation of limited dependent variable spatial autoregressive models. Geogr Anal 32(1):19-35

LeSage JP, Pace RK (2007) A matrix exponential spatial specification. J Econ 140(1):190-214

LeSage JP, Fischer MM (2008) Spatial growth regression: model specification, estimation and interpretation. Spat Econ Anal 3(3):275-304

LeSage JP, Pace RK (2008) Spatial econometric modeling of origin-destination flows. J Reg Sci 48(5):914-967

LeSage JP, Pace RK (2009) Introduction to spatial econometrics. CRC Press, Taylor \& Francis Group, Boca Raton [FL]

LeSage JP, Parent O (2007) Bayesian model averaging for spatial econometric models. Geogr Anal 39(3):241-267

Lin X, LeSage JP (2010) A Matlab-based spatial data analysis toolbox. J Geo Sys 12(1):69-87

Ord JK (1975) Estimation methods for models of spatial interaction. J Am Stat Assoc 70(349):120-26

Rey SJ, Anselin L (2000) Regional science publication patterns in the 1990s. Int Reg Sci Rev 23(4):323-344

Smith TE, LeSage JP (2004) A Bayesian probit model with spatial dependencies. In: LeSage JP, Pace RK (eds) Spatial and spatiotemproal econometrics. Advances in Econometrics Volume 18, pp. 127-160. Elsevier, Amsterdam 